\title{
A STUDY OF THE GENERALIZED CHRISTOFFEL FUNCTIONS WITH APPLICATIONS*
}

\author{
E. BERRIOCHOA ${ }^{\dagger}$, A. CACHAFEIRO $\ddagger$, AND F. MARCELLÁN $\S$
}

To Richard Askey on his 65th birthday

\begin{abstract}
In this paper we solve the problem of minimizing the norm of polynomials of degree less than or equal to $n$, verifying linear restrictions. This case extends in a natural way a problem studied by Grenander and Rosenblatt. We obtain algebraic properties of the solution which enables us to compute it and we present some applications.
\end{abstract}

1. Introduction. Let $\mu$ be a finite positive Borel measure on $[0,2 \pi)$ with an infinite set as support. In the linear space $\mathbb{P}$ of algebraic polynomials with complex coefficients we consider the inner product:

$$
<P, Q>=\int_{0}^{2 \pi} P\left(e^{i \theta}\right) \overline{Q\left(e^{i \theta}\right)} d \mu(\theta), \quad \forall P, Q \in \mathbb{P} .
$$

We denote the Gram matrix of $\left\{z^{k}\right\}_{k=0}^{n}$ by $M_{n+1}=\left[<z^{i}, z^{j}>\right]_{\substack{i=0, \ldots, n \\ j=0, \ldots, n}}$ and the corresponding moments by $c_{i-j}=\left\langle z^{i}, z^{j}>i, j=0,1,2, \cdots\right.$. We denote $\|P\|^{2}=$ $<P, P>$.

By applying the Gram-Schmidt process to the sequence $\left\{z^{n}\right\}_{n>0}$, we can obtain the sequence of orthonormal polynomials $\left\{\varphi_{n}\right\}$, such that

$$
\varphi_{n}(z)=k_{n} z^{n}+\text { lower degree terms }
$$

with $k_{n}>0$.

Also we consider the sequence of monic orthogonal polynomials, $\left\{\phi_{n}\right\}_{n \geq 0}$, defined by $\phi_{n}=\frac{\varphi_{n}}{k_{n}}$.

If we denote by $\mathbb{P}_{n}$ the linear subspace of polynomials of degree at most $n$, it is known that there exists a unique polynomial function of two variables, $K_{n}(z, y)$, and degree $n$ in $z$ and $y$, such that it has the reproducing property on $\mathbb{P}_{n}$, that is $\left\langle K_{n}(z, y), P(z)>=\overline{P(y)} \forall P \in \mathbb{P}_{n}\right.$. The function $K_{n}(z, y)$ is called the $n$th reproducing kernel and $K_{n}(z, y)=\sum_{k=0}^{n} \varphi_{k}(z) \overline{\varphi_{k}(y)}$, (see [Aro]). In the theory of orthogonal polynomials, it is well-known that the monic orthogonal polynomials and the kernels satisfy some extremal conditions, (see [VAss]):

*Received June 1, 1998, revised Oct. 22, 1998.

†Departamento de Matemática Aplicada, Fac. Ciencias, Universidad de Vigo, Spain (esnaola @setei.uvigo.es). The research of this author was supported by Dirección General de Enseñanza Superior (DGES) of Spain PB96-0344.

$\ddagger$ Departamento de Matemática Aplicada, E. T. S. I. I., Universidad de Vigo, Spain (acachafe @dma.uvigo.es). The research of this author was supported by Dirección General de Enseñanza Superior (DGES) of Spain PB96-0344.

$\S$ Departamento de Matemáticas, Escuela Politécnica Superior, Universidad Carlos III, Madrid, Spain (pacomarc@ing.uc3m.es). The research of this author was supported by Dirección General de Enseñanza Superior (DGES) of Spain PB96-0120-C03-01. 
1. $\min _{P \in \mathbb{P}_{n}, \frac{P^{(n)}(0)}{n !}=1}\left\{\|P\|^{2}\right\}=\frac{1}{k_{n}^{2}}$ and it is attained at $P=\phi_{n}$.

2. If $z_{0}$ is an arbitrary point in the complex plane, then: $\min _{P \in \mathbb{P}_{n}, P\left(z_{0}\right)=1}\left\{\|P\|^{2}\right\}$ $=\frac{1}{K_{n}\left(z_{0}, z_{0}\right)}$ and it is attained at $P(z)=\frac{K_{n}\left(z, z_{0}\right)}{K_{n}\left(z_{0}, z_{0}\right)}$.

In the same way, we can consider the minimal problems corresponding to intermediate cases, see ([Be]). For $0 \leq r \leq n \min _{P \in \mathbb{P}_{n}, P^{(r)}\left(z_{0}\right)=1}\|P\|^{2}=\frac{1}{K_{n}^{(r, r)}\left(z_{0}, z_{0}\right)}$ and it is attained at $P(z)=\frac{K_{n}^{(0, r)}\left(z, z_{0}\right)}{K_{n}^{(r, r)}\left(z_{0}, z_{0}\right)}$, where we denote by $K_{n}^{(r, r)}\left(z_{0}, z_{0}\right)=\sum_{k=0}^{n}\left|\varphi_{k}^{(r)}\left(z_{0}\right)\right|^{2}$ and $K_{i 2}^{(0, r)}\left(z, z_{0}\right)=\sum_{k=0}^{n} \varphi_{k}(z) \overline{\varphi_{k}^{(r)}\left(z_{0}\right)}$.

Arother extremal characterization for these polynomials is the following:

$$
\min _{P \in \mathbb{P}_{n}}\left\{\|P\|^{2}-2 \Re P^{(r)}\left(z_{0}\right)\right\}=-K_{n}^{(r, r)}\left(z_{0}, z_{0}\right)
$$

and it is attained at $P(z)=K_{n}^{(0, r)}\left(z, z_{0}\right)$. We are going to prove this property in the next section (Theorem 3 ) in a more general situation.

Thus, if we introduce the Christoffel function $w_{n}(d \mu)$, defined by $w_{n}(d \mu)(z)=$ $\frac{1}{K_{n}(z, z)}$, then it holds that the minimum value in property 2 is $w_{n}(d \mu)\left(z_{0}\right)$, (see [Nev1]).

In the same way, using generalized Christoffel Functions $w_{n}(d \mu, m)$, one can write $\min _{P \in \mathbb{P}_{n}, P\left(z_{0}\right)=1, P^{\prime}\left(z_{0}\right)=0, \cdots, P^{(m)}\left(z_{0}\right)=0}\|P\|^{2}=w_{n}(d \mu, m)\left(z_{0}\right)$, (see [Nev2]).

A more general case is studied in [GrRo], where the following problem is solved. Find the minimum:

$\min _{p \in \mathbb{P}_{n}}\|P\|^{2}$, with the restrictions $P^{(k)}\left(\alpha_{j}\right)=\beta_{j}^{k}$ for $j=1, \cdots, m, k=0, \cdots, n_{j}$

where the $\alpha_{j}$ are different points in the closed unit circle and the $\beta_{j}^{k}$ do not all vanish. The explicit solution of this minimum problem is given in terms of determinants involving the kernel function and its derivatives. Moreover, they also say that the results are valid with appropriate modifications when the restrictions are of the form: $P^{(k)}\left(\alpha_{j}\right)=\beta_{j}^{k}, k \in S_{j}, j=1, \cdots, n_{j}$ where $S_{j}$ is a finite set of nonnegative integers. All these problems appear in the theory of linear prediction of stationary discrete-time stochastic processes. In fact they allow to construct best predictors.

The preceding problems can be extended in a natural way to the following.

Let $f_{1}, \cdots, f_{m}$ be $m$ linear functionals in $\mathbb{P}$, such that $\left.f_{j}\right|_{\mathbb{P}_{n}} \not \equiv 0$. If $f_{1}, \cdots, f_{m}$ are linearly independent, find the minimum of the quadratic form $\|P\|^{2}$ given by the matrix $M_{n+1}$, under the restrictions $f_{j}(P)=a_{j}, j=1, \cdots, m$.

Our aim is to study this last problem. In Section 2 we solve the problem with only one linear restriction. We obtain algebraic properties of the solution which enables us to compute it. In Section 3 we solve the general problem and in Section 4 we present some applications. 
2. Generalized christoffel functions. Throughout this section, let $f$ be a linear functional on $\mathbb{P}$, such that $\left.f\right|_{\mathbb{P}_{n}} \not \equiv 0, \forall n$. We are going to analyze the following problem. Find $\min \left\{\|P\|^{2}: P \in \mathbb{P}\right.$ and $\left.f(P)=1\right\}$. In order to do this, we introduce, in the following definition, a family of polynomials.

DeFinition 1. We denote by ${ }_{f} \psi_{n}$ the polynomial in $\mathbb{P}_{n}$, such that $f\left({ }_{f} \psi_{n}\right)=1$, and $<{ }_{f} \psi_{n}, P>=0$ for all $P \in \mathbb{P}_{n}$, such that $f(P)=0$.

It is clear that, for each $n$, there exists a unique polynomial ${ }_{f} \psi_{n}$ verifying the preceding definition. Taking into account $\mathbb{P}_{n}=\operatorname{Ker}\left(\left.f\right|_{\mathbb{P}_{n}}\right) \bigoplus^{\perp} \operatorname{span}\{q\}$ for some $q \in \mathbb{P}_{n}$, such that $f(q) \neq 0$, then we choose ${ }_{f} \psi_{n}=\frac{q}{f(q)}$. The polynomials in the above definition were introduced in [Be], and the following minimal property can be deduced.

THEOREM 1. The minimum of the norm, taken over all polynomials $q \in \mathbb{P}_{n}$, with the constraint $f(q)=1$, is attained for $q={ }_{f} \psi_{n}$. The minimum is equal to $\left\|{ }_{f} \psi_{n}\right\|$.

Proof. See $[\mathrm{Be}]$.

The polynomials satisfying the above extremal conditions can be related with the generalized Christoffel functions, see [Nev2]. Next we are going to find explicit expressions in order to compute them.

THEOREM 2. The sequence $\left\{{ }_{f} \psi_{n}\right\}_{n \geq 0}$ satisfies the following forward recurrence relation:

$$
{ }_{f} \psi_{n+1}={ }_{f} \beta_{n}\left({ }_{f} \psi_{n}+{ }_{f} \alpha_{n} \phi_{n+1}\right) \quad n \geq 0,
$$

with ${ }_{f} \psi_{0}=\left(\left.f\right|_{\mathbb{P}_{0}}\right)^{-1}(1)$. The coefficients ${ }_{f} \alpha_{n}$ and ${ }_{f} \beta_{n}$ are complex numbers, ${ }_{f} \beta_{n} \neq 0$ given by:

$$
{ }_{f} \alpha_{n}=\frac{\overline{f\left(\phi_{n+1}\right)}\left\|_{f} \psi_{n}\right\|^{2}}{\left\|\phi_{n+1}\right\|^{2}} \text { and }{ }_{f} \beta_{n}=\left(1+\frac{\left|f\left(\phi_{n+1}\right)\right|^{2}\left\|{ }_{f} \psi_{n}\right\|^{2}}{\left\|\phi_{n+1}\right\|^{2}}\right)^{-1} .
$$

Proof. Since

$$
\mathbb{P}_{n+1}=\mathbb{P}_{n} \oplus^{\perp} \operatorname{span}\left\{\phi_{n+1}\right\}=\operatorname{span}\left\{{ }_{f} \psi_{n}\right\} \oplus^{\perp} \operatorname{Ker}\left(\left.f\right|_{\mathbb{P}_{n}}\right) \oplus^{\perp} \operatorname{span}\left\{\phi_{n+1}\right\},
$$

we introduce the polynomial ${ }_{f} \psi_{n}+{ }_{f} \alpha_{n} \phi_{n+1}$, with ${ }_{f} \alpha_{n}$ a complex number.

If $q \in \mathbb{P}_{n}$ and $f(q)=0$, then $\left.<{ }_{f} \psi_{n}+{ }_{f} \alpha_{n} \phi_{n+1}, q\right\rangle=\left\langle{ }_{f} \psi_{n}, q\right\rangle+_{f} \alpha_{n}\left\langle\phi_{n+1}, q\right\rangle$ $=0$. Let $q=z^{n+1}+$ lower degree terms, such that $f(q) \neq 0$, and impose that $<{ }_{f} \psi_{n}+{ }_{f} \alpha_{n} \phi_{n+1}, q>=0$. This yields

$$
{ }_{f} \alpha_{n}=-\frac{<{ }_{f} \psi_{n}, q>}{\left\|\phi_{n+1}\right\|^{2}} .
$$

If $<_{f} \psi_{n}, q>=0$ for every $q=z^{n+1}+$ lower degree terms, such that $f(q)=0$ then ${ }_{f} \psi_{n}={ }_{f} \psi_{n+1}$ and (1) is true. In other case ${ }_{f} \alpha_{n} \neq 0$. 
On. the other hand, it holds that ${ }_{f} \psi_{n}+_{f} \alpha_{n} \phi_{n+1} \neq 0$ because $<_{f} \psi_{n}+_{f} \alpha_{n} \phi_{n+1},{ }_{f} \psi_{n}>$ $=\left\|_{f} \psi_{n}\right\|^{2} \neq 0$. Moreover, it is easy to see that $f\left({ }_{f} \psi_{n}+{ }_{f} \alpha_{n} \phi_{n+1}\right) \neq 0$. Indeed, if we assume that $f\left({ }_{f} \psi_{n}+_{f} \alpha_{n} \phi_{n+1}\right)=0$, we deduce $f\left(\phi_{n+1}\right)=\frac{1}{{ }_{f} \alpha_{n}}$. Then $\phi_{n+1} \notin \operatorname{Ker}\left(\left.f\right|_{\mathbb{P}_{n+1}}\right)$ and so $\phi_{n+1}=A_{f} \psi_{n+1}$ for some $A \in \mathbb{C}$. Therefore $<\phi_{n+1},_{f} \psi_{n}+{ }_{f} \alpha_{n} \phi_{n+1}>=0$, which implies $\overline{f^{\alpha_{n}}}\left\|\phi_{n+1}\right\|^{2}=0$, and ${ }_{f} \alpha_{n}=0$, leading a contradiction. (1).

Thus there exists ${ }_{f} \beta_{n} \neq 0$ such that ${ }_{f} \beta_{n}\left({ }_{f} \psi_{n}+{ }_{f} \alpha_{n} \phi_{n+1}\right)={ }_{f} \psi_{n+1}$, which proves

Next we obtain explicit expressions for the coefficients. By applying $f$ in (1) we obtain

$$
1={ }_{f} \beta_{n}\left(1+{ }_{f} \alpha_{n} f\left(\phi_{n+1}\right)\right) \Rightarrow{ }_{f} \beta_{n}=\frac{1}{1+{ }_{f} \alpha_{n} f\left(\phi_{n+1}\right)} .
$$

From (4), ${ }_{f} \alpha_{n}=-\frac{\left\langle{ }_{f} \psi_{n}, q\right\rangle}{\left\|\phi_{n+1}\right\|^{2}}$ with $q=z^{n+1}+$ lower degree terms and $f(q)=0$. Taking into account (3) we have $q=A_{f} \psi_{n}+R+\phi_{n+1}$, with $A \in \mathbb{C}$ and $R \in \operatorname{Ker}\left(\left.f\right|_{\mathbb{P}_{n}}\right)$. Since $f(q)=0$, then $A=-f\left(\phi_{n+1}\right)$. Therefore

$$
{ }_{f} \alpha_{n}=-\frac{<_{f} \psi_{n},-f\left(\phi_{n+1}\right)_{f} \psi_{n}+R+\phi_{n+1}>}{\left\|\phi_{n+1}\right\|^{2}}=\frac{\overline{f\left(\phi_{n+1}\right)}\left\|_{f} \psi_{n}\right\|^{2}}{\left\|\phi_{n+1}\right\|^{2}}
$$

from which, combined with (5), it follows (2).

Corollary 1. The sequence $\left\{{ }_{f} \psi_{n}\right\}_{n \geq 0}$ verifies the following backward recurrence relation:

$$
{ }_{f} \psi_{n}=\left({ }_{f} \beta_{n}\right)^{-1}\left({ }_{f} \psi_{n+1}-{ }_{f} \alpha_{n}{ }_{f} \beta_{n} \phi_{n+1}\right)
$$

Proof. It is an immediate consequence of (1). Moreover, when $f$ is the linear functional defined by $f(P)=P(\dot{0})$, then ${ }_{f} \psi_{n}=\phi_{n}^{*}$, and therefore relation (6) is the well-known Szegö's backward recurrence relation, (see [Sze]).

Next we develop another way for the computation of the sequence $\left\{{ }_{f} \psi_{n}\right\}_{n \geq 0}$. Following the usual notation for the kernels we can write:

$$
K_{n}^{(-, f)}=\sum_{k=0}^{n} \varphi_{k} \overline{f\left(\varphi_{k}\right)}, K_{n}^{(f,-)}=\overline{K_{n}^{(-, f)}}, \text { and } K_{n}^{(f, f)}=\sum_{k=0}^{n}\left|f\left(\varphi_{k}\right)\right|^{2} .
$$

Taking into account that $\min _{q \in \mathbb{P}_{n}, f(q)=1}\|q\|^{2}=\left(K_{n}^{(f, f)}\right)^{-1}$ and the fact that it is attained for $q=\frac{K_{n}^{(-, f)}}{K_{n}^{(f, f)}}$, (see [Be]), from Theorem 1 we deduce that ${ }_{f} \psi_{n}=\frac{K_{n}^{(-, f)}}{K_{n}^{(f, f)}}$ and $\left\|{ }_{f} \psi_{n}\right\|^{2}=\frac{1}{K_{n}^{(f, f)}}$. Therefore we can obtain ${ }_{f} \psi_{n}$ by computing $K_{n}^{(-, f)}$ and $K_{n}^{(f, f)}$, and it holds that:

$$
K_{n}^{(-, f)}=\left(\overline{f(1)}, \cdots, \overline{f\left(y^{n}\right)}\right)\left(M_{n+1}^{-1}\right)^{T}\left(1, \cdots, z^{n}\right)^{T}
$$

as well as

$$
K_{n}^{(f, f)}=\left(\overline{f(1)}, \cdots, \overline{f\left(y^{n}\right)}\right)\left(M_{n+1}^{-1}\right)^{T}\left(f(1), \cdots, f\left(z^{n}\right)\right)^{T}
$$


Some other properties concerning the polynomials ${ }_{f} \psi_{n}$ can be deduced.

THEOREM 3 .

$$
\min _{P \in \mathbb{P}_{n}}\left\{\|P\|^{2}-2 \Re f(P)\right\}=-K_{n}^{(f, f)}
$$

and it is attained for $P=K_{n}^{(-, f)}=\frac{{ }_{f} \psi_{n}}{\left\|{ }_{f} \psi_{n}\right\|^{2}}$

Proof. Let $P(z)=\sum_{k=0}^{n} \lambda_{k} \varphi_{k}(z)$. Then:

$$
\begin{array}{r}
\|P\|^{2}-2 \Re f(P)=\sum_{k=0}^{n}\left|\lambda_{k}\right|^{2}-2 \Re\left(\sum_{k=0}^{n} \lambda_{k} f\left(\varphi_{k}\right)\right)= \\
\sum_{k=0}^{n}\left|\lambda_{k}\right|^{2}-\sum_{k=0}^{n} \lambda_{k} f\left(\varphi_{k}\right)-\sum_{k=0}^{n} \overline{\lambda_{k} f\left(\varphi_{k}\right)}=\sum_{k=0}^{n}\left|\lambda_{k}-\overline{f\left(\varphi_{k}\right)}\right|^{2}-\sum_{k=0}^{n}\left|f\left(\varphi_{k}\right)\right|^{2} .
\end{array}
$$

Thus $\min _{P \in \mathbb{P}_{n}}\left\{\|P\|^{2}-2 \Re f(P)\right\}=-K_{n}^{(f, f)}$ and it is attained for $P(z)=\sum_{k=0}^{n} \overline{f\left(\varphi_{k}\right)} \varphi_{k}(z)$ $=K_{n}^{(-, f)}$.

THEOREM 4. Let $f_{1}, \cdots, f_{m}$ be $m$ linear functionals in $\mathbb{P}_{n}$ which are linearly independent. Then the polynomials $f_{1} \psi_{n}, \cdots, f_{m} \psi_{n}$ are linearly independent.

Proof. Let $\sum_{j=1}^{m} A_{j f_{j}} \psi_{n}=0$, that is, $\sum_{j=1}^{m} A_{j} \frac{K_{n}^{\left(-, f_{j}\right)}}{K_{n}^{\left(f_{j}, f_{j}\right)}}=0$. Taking into account (7), we have:

$$
A_{1} \sum_{k=0}^{n} \frac{\overline{f_{1}\left(\varphi_{k}\right)}}{K_{n}^{\left(f_{1}, f_{1}\right)}} \varphi_{k}+\cdots+A_{m} \sum_{k=0}^{n} \frac{\overline{f_{m}\left(\varphi_{k}\right)}}{K_{n}^{\left(f_{m}, f_{m}\right)}} \varphi_{k}=0
$$

Since $\left\{\varphi_{k}\right\}_{k=0}^{n}$ are linearly independent, we get

$$
\left(\frac{\overline{A_{1}}}{K_{n}^{\left(f_{1}, f_{1}\right)}} f_{1}+\cdots+\frac{\overline{A_{m}}}{K_{n}^{\left(f_{m}, f_{m}\right)}} f_{m}\right)\left(\varphi_{k}\right)=0, k=0, \cdots, n
$$

which yields $\sum_{j=1}^{m} \frac{\overline{A_{j}}}{K_{n}^{\left(f_{j}, f_{j}\right)}} f_{j}=0$ and therefore $A_{j}=0$ for $j=1, \cdots, m$.

\section{Minimizing quadratic forms with linear restrictions.}

THEOREM 5. Let $m$ and $n$ be nonnegative integer numbers, with $m \leq n$. Let $f_{1}, \cdots, f_{m}$ be $m$ linearly independent linear functionals in $\mathbb{P}$, such that $\left.\bar{f}_{j}\right|_{\mathbb{P}_{n}} \not \equiv 0$ $\forall j=1, \cdots, m$, and let $a_{1}, \cdots, a_{m}$ be $m$ complex numbers. Then:

1. There exists $q \in \mathbb{P}_{n}$ such that $f_{j}(q)=a_{j}$ for $j=1, \cdots, m$ if and only if there exists $p \in \operatorname{span}\left\{f_{1} \psi_{n}, \cdots, f_{m} \psi_{n}\right\}$ such that $f_{j}(p)=a_{j}$ for $j=1, \cdots, m$.

2. If there exists $p \in \operatorname{span}\left\{f_{1} \psi_{n}, \cdots, f_{m} \psi_{n}\right\}$ such that $f_{j}(p)=a_{j}$ for $j=$ $1, \cdots, m$ then $p$ is uniquely determined. 


\section{Proof.}

1. Let $q \in \mathbb{P}_{n}$ such that $f_{j}(q)=a_{j}$ for $j=1, \cdots, m$. It is clear that $q$ may be written as:

$q=p+l$, with $p \in \operatorname{span}\left\{f_{1} \psi_{n}, \cdots, f_{m} \psi_{n}\right\}$ and $l \in \operatorname{span}\left\{f_{1} \psi_{n}, \cdots, f_{m} \psi_{n}\right\}^{\perp}$.

'Taking into account that $l \in \operatorname{span}\left\{f_{j} \psi_{n}\right\}^{\perp}$ for $j=1, \cdots, m$, then $f_{j}(l)=0$ for $j=1, \cdots, m$. Therefore $f_{j}(p)=f_{j}(q-l)=a_{j}$ for $j=1, \cdots, m$.

The converse is straightforward.

2. Let $p_{1}, p_{2} \in \operatorname{span}\left\{f_{1} \psi_{n}, \cdots, f_{m} \psi_{n}\right\}$ verifying $f_{j}\left(p_{1}\right)=f_{j}\left(p_{2}\right)=a_{j}$ for $j=$ $1, \cdots, m$. Since $f_{j}\left(p_{1}-p_{2}\right)=0$ for $j=1, \cdots, m$, then $p_{1}-p_{2} \epsilon$ $\operatorname{span}\left\{f_{1} \psi_{n}, \cdots, f_{m} \psi_{n}\right\}^{\perp}$. On the other hand, $p_{1}-p_{2} \in \operatorname{span}\left\{f_{1} \psi_{n}, \cdots, f_{m} \psi_{n}\right\}$ and therefore $p_{1}=p_{2}$.

Notice that if there exists $q \in \mathbb{P}_{n}$ such that $f_{j}(q)=a_{j}$ for $j=1, \cdots, m$, then all those solutions can be written $p+\operatorname{span}\left\{f_{1} \psi_{n}, \cdots, f_{m} \psi_{n}\right\}^{\perp}$, with $p$ the unique polynomial in $\operatorname{span}\left\{f_{1} \psi_{n}, \cdots, f_{m} \psi_{n}\right\}$ satisfying $f_{j}(p)=a_{j}$ for $j=1, \cdots, m$.

THEOREM 6. Let $m$ and $n$ be nonnegative integer numbers, with $m \leq n$. Let $f_{1}, \cdots, f_{m}$ be $m$ linearly independent linear functionals in $\mathbb{P}$ such that $\left.f_{j}\right|_{\mathbb{P}_{n}} \not \equiv 0 \forall j=$ $1, \cdots, m$ and let $a_{1}, \cdots, a_{m}$ be $m$ complex numbers. If there exists $q \in \mathbb{P}_{n}^{n}$ such that $f_{j}(q)=a_{j}$ for $j=1, \cdots, m$, then:

$$
\min _{\substack{p \in \mathbb{P}_{n} \\ f_{j}(p)=a_{j}, j=1, \cdots, m}}\|p\|^{2}
$$

is attained at a unique polynomial $p \in \operatorname{span}\left\{f_{1} \psi_{n}, \cdots, f_{m} \psi_{n}\right\}$ satisfying $f_{j}(p)=a_{j}$ for $j=1, \cdots, m$.

Proof. If $q \in \mathbb{P}_{n}$ satisfies $f_{j}(p)=a_{j}$ for $j=1, \cdots, m$, we have proved that $q=p+l$, with $p \in \operatorname{span}\left\{f_{1} \psi_{n}, \cdots, f_{m} \psi_{n}\right\}, l \in \operatorname{span}\left\{f_{1} \psi_{n}, \cdots, f_{m} \psi_{n}\right\}^{\perp}$ and $f_{j}(p)=a_{j}$ for $j=1, \cdots, m$.

Therefore, $\|q\|^{2}=\|p\|^{2}+\|l\|^{2} \geq\|p\|^{2}$. $\square$

THEOREM 7. Let $m$ and $n$ be nonnegative integer numbers, with $m \leq n$. Let $f_{1}, \cdots, f_{m}$ be $m$ linearly independent linear functionals in $\mathbb{P}$ such that $\left.f_{j}\right|_{\mathbb{P}_{n}} \not \equiv 0 \forall j=$ $1, \cdots, m$ and let $a_{1}, \cdots, a_{m}$ be $m$ complex numbers. Then:

$$
\min _{\substack{p \in \mathbb{P}_{n} \\
f_{j}(p)=a_{j}, j=1, \cdots, m}}\|p\|^{2}=-\frac{\left|\begin{array}{cccc}
K_{n}^{\left(f_{1}, f_{1}\right)} & \cdots & K_{n}^{\left(f_{1}, f_{m}\right)} & \overline{a_{1}} \\
\vdots & \ddots & \vdots & \vdots \\
K_{n}^{\left(f_{m}, f_{1}\right)} & \cdots & K_{n}^{\left(f_{m}, f_{m}\right)} & \overline{a_{m}} \\
a_{1} & \cdots & a_{m} & 0
\end{array}\right|}{\left|\begin{array}{cccc}
K_{n}^{\left(f_{1}, f_{1}\right)} & \cdots & K_{n}^{\left(f_{1}, f_{m}\right)} \\
\vdots & \ddots & \vdots \\
K_{n}^{\left(f_{m}, f_{1}\right)} & \cdots & K_{n}^{\left(f_{m}, f_{m}\right)}
\end{array}\right|}
$$


The minimum is attained at:

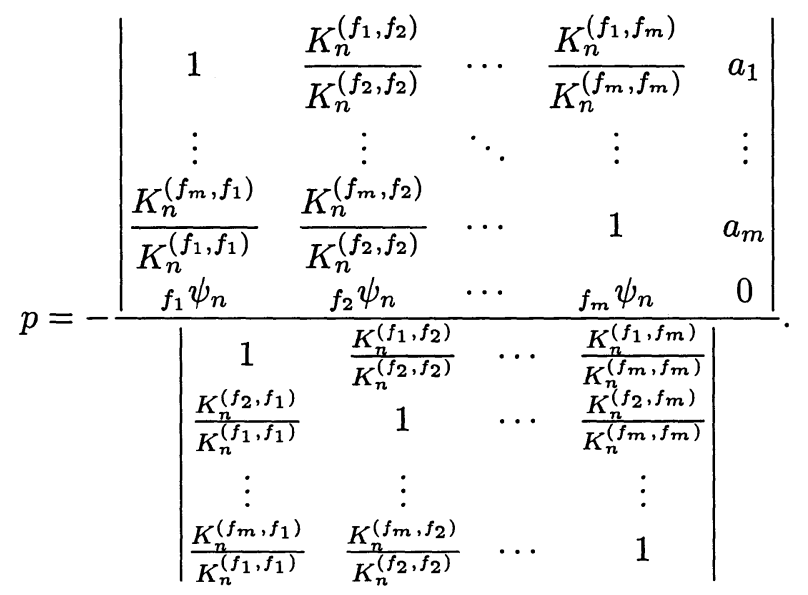

Proof. Under our hypotheses there exists $p \in \operatorname{span}\left\{f_{1} \psi_{n}, \cdots, f_{m} \psi_{n}\right\}$ satisfying $f_{i}(p)=a_{i}$ for $i=1, \cdots, m$. Indeed, let $p=\sum_{j=1}^{m} A_{j f_{j}} \psi_{n}$. If we assume that $f_{i}(p)=a_{i}$ for $i=1, \cdots, m$, then we have:

$$
a_{i}=\sum_{j=1}^{m} A_{j} f_{i}\left(f_{j} \psi_{n}\right) \quad i=1, \cdots, m
$$

that is,

$$
\left(\begin{array}{c}
a_{1} \\
\vdots \\
a_{m}
\end{array}\right)=\left(\begin{array}{cccc}
1 & f_{1}\left(f_{2} \psi_{n}\right) & \cdots & f_{1}\left(f_{m} \psi_{n}\right) \\
f_{2}\left(f_{1} \psi_{n}\right) & 1 & \cdots & f_{2}\left(f_{m} \psi_{n}\right) \\
\vdots & \vdots & & \vdots \\
f_{m}\left(f_{1} \psi_{n}\right) & f_{m}\left(f_{2} \psi_{n}\right) & \cdots & 1
\end{array}\right)\left(\begin{array}{c}
A_{1} \\
\vdots \\
A_{m}
\end{array}\right)
$$

or, equivalently, we can write

$$
\left(\begin{array}{c}
a_{1} \\
\vdots \\
a_{m}
\end{array}\right)=\left(\begin{array}{cccc}
1 & \frac{K_{n}^{\left(f_{1}, f_{2}\right)}}{K_{n}^{\left(f_{2}, f_{2}\right)}} & \cdots & \frac{K_{n}^{\left(f_{1}, f_{m}\right)}}{K_{n}^{\left(f_{m}, f_{m}\right)}} \\
\frac{K_{n}^{\left(f_{2}, f_{1}\right)}}{K_{n}^{\left(f_{1}, f_{1}\right)}} & 1 & \cdots & \frac{K_{n}^{\left(f_{2}, f_{m}\right)}}{K_{n}^{\left(f_{m}, f_{m}\right)}} \\
\vdots & \vdots & & \vdots \\
\frac{K_{n}^{\left(f_{m}, f_{1}\right)}}{K_{n}^{\left(f_{1}, f_{1}\right)}} & \frac{K_{n}^{\left(f_{m}, f_{2}\right)}}{K_{n}^{\left(f_{2}, f_{2}\right)}} & \cdots & 1
\end{array}\right)\left(\begin{array}{c}
A_{1} \\
\vdots \\
A_{m}
\end{array}\right) .
$$

Next we prove that $\mathbf{K}_{n}=\left(K_{n}^{\left(f_{i}, f_{j}\right)}\right)_{\substack{i=1, \cdots, m \\ j=1, \cdots, m}}$ is a nonsingular matrix. Indeed $\mathbf{K}_{n}=$

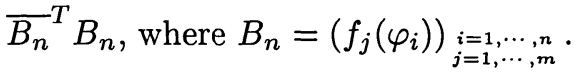

Let $X=\left(x_{1}, \cdots, x_{m}\right) \in \mathbb{C}^{m}$ and assume that $B_{n} X^{T}=0$. Then $\sum_{i=1}^{m} x_{i} f_{i}=0$, and taking into account that the linear functionals $f_{i}(i=1, \cdots, m)$ are linearly independent, we obtain $X=0$, which implies that rank of $B_{n}$ is $m$. Thus, rank $\mathbf{K}_{n}=m$. 
Therefore the coefficients $A_{j}(j=1, \cdots, m)$ are given by:

$$
\left(\begin{array}{c}
A_{1} \\
\vdots \\
A_{m}
\end{array}\right)=\left(\begin{array}{cccc}
1 & \frac{K_{n}^{\left(f_{1}, f_{2}\right)}}{K_{n}^{\left(f_{2}, f_{2}\right)}} & \cdots & \frac{K_{n}^{\left(f_{1}, f_{m}\right)}}{K_{n}^{\left(f_{m}, f_{m}\right)}} \\
\frac{K_{n}^{\left(f_{2}, f_{1}\right)}}{K_{n}^{\left(f_{1}, f_{1}\right)}} & 1 & \cdots & \frac{K_{n}^{\left(f_{2}, f_{m}\right)}}{K_{n}^{\left(f_{m}, f_{m}\right)}} \\
\vdots & \vdots & & \vdots \\
\frac{K_{n}^{\left(f_{m}, f_{1}\right)}}{K_{n}^{\left(f_{1}, f_{1}\right)}} & \frac{K_{n}^{\left(f_{m}, f_{2}\right)}}{K_{n}^{\left(f_{2}, f_{2}\right)}} & \cdots & 1
\end{array}\right)^{-1} \quad\left(\begin{array}{c}
a_{1} \\
\vdots \\
a_{m}
\end{array}\right)
$$

and applying the preceding Theorems 5 and 6 we obtain the result. Indeed

$$
\begin{aligned}
& p=\left(\begin{array}{lll}
f_{1} \psi_{n} & \cdots & f_{m} \psi_{n}
\end{array}\right)\left(\begin{array}{c}
A_{1} \\
\vdots \\
A_{m}
\end{array}\right) \\
& =\left(\begin{array}{lll}
\frac{K_{n}^{\left(-, f_{1}\right)}}{K_{n}^{\left(f_{1}, f_{1}\right)}} & \cdots & \frac{K_{n}^{\left(-, f_{m}\right)}}{K_{n}^{\left(f_{m}, f_{m}\right)}}
\end{array}\right)\left(\begin{array}{cccc}
1 & \frac{K_{n}^{\left(f_{1}, f_{2}\right)}}{K_{n}^{\left(f_{2}, f_{2}\right)}} & \cdots & \frac{K_{n}^{\left(f_{1}, f_{m}\right)}}{K_{n}^{\left(f_{m}, f_{m}\right)}} \\
\frac{K_{n}^{\left(f_{2}, f_{1}\right)}}{K_{n}^{\left(f_{1}, f_{1}\right)}} & 1 & \cdots & \frac{K_{n}^{n}\left(f_{2}, f_{m}\right)}{K_{n}^{\left(f_{m}, f_{m}\right)}} \\
\vdots & \vdots & & \vdots \\
\frac{K_{n}^{\left(f_{m}, f_{1}\right)}}{K_{n}^{\left(f_{1}, f_{1}\right)}} & \frac{K_{n}^{\left(f_{m}, f_{2}\right)}}{K_{n}^{\left(f_{2}, f_{2}\right)}} & \cdots & 1
\end{array}\right)^{-1}\left(\begin{array}{c}
a_{1} \\
\vdots \\
a_{m}
\end{array}\right) \\
& =\left(\begin{array}{lll}
K_{n}^{\left(-, f_{1}\right)} & \cdots & K_{n}^{\left(-, f_{m}\right)}
\end{array}\right)\left(\begin{array}{cccc}
K_{n}^{\left(f_{1}, f_{1}\right)} & K_{n}^{\left(f_{1}, f_{2}\right)} & \cdots & K_{n}^{\left(f_{1}, f_{m}\right)} \\
K_{n}^{\left(f_{2}, f_{1}\right)} & K_{n}^{\left(f_{2}, f_{2}\right)} & \cdots & K_{n}^{\left(f_{2}, f_{m}\right)} \\
\vdots & \vdots & \ddots & \vdots \\
K_{n}^{\left(f_{m}, f_{1}\right)} & K_{n}^{\left(f_{m}, f_{2}\right)} & \cdots & K_{n}^{\left(f_{m}, f_{m}\right)}
\end{array}\right)^{-1}\left(\begin{array}{c}
a_{1} \\
\vdots \\
a_{m}
\end{array}\right) \\
& =\sum_{k=1}^{m} c_{k} \tilde{\psi}_{n, k},
\end{aligned}
$$

where the polynomials $\tilde{\psi}_{n, k}$ are given by the following expressions:

$$
\tilde{\psi}_{n, k}=\frac{\left|\begin{array}{ccc}
K_{n}^{\left(f_{1}, f_{1}\right)} & \cdots & K_{n}^{\left(f_{1}, f_{m}\right)} \\
\vdots & \vdots & \vdots \\
K_{n}^{\left(f_{k-1}, f_{1}\right)} & \cdots & K_{n}^{\left(f_{k-1}, f_{m}\right)} \\
K_{n}^{\left(-, f_{1}\right)} & \cdots & K_{n}^{\left(-, f_{m}\right)} \\
K_{n}^{\left(f_{k+1}, f_{1}\right)} & \cdots & K_{n}^{\left(f_{k+1}, f_{m}\right)} \\
\vdots & \vdots & \vdots \\
K_{n}^{\left(f_{m}, f_{1}\right)} & \cdots & K_{n}^{\left(f_{m}, f_{m}\right)}
\end{array}\right|}{\operatorname{det} \mathbf{K}_{n}}
$$

It is clear that $\tilde{\psi}_{n, k}$ satisfies the condition $f_{i}\left(\tilde{\psi}_{n, k}\right)=\delta_{i, k}$. Finally,

$$
\|p\|^{2}=\sum_{k, j=1}^{m} a_{k} \overline{a_{j}}<\tilde{\psi}_{n, k}, \tilde{\psi}_{n, j}>=-\frac{\left|\begin{array}{cccc}
K_{n}^{\left(f_{1}, f_{1}\right)} & \ldots & K_{n}^{\left(f_{1}, f_{m}\right)} & \overline{a_{1}} \\
\vdots & \ddots & \vdots & \vdots \\
K_{n}^{\left(f_{m}, f_{1}\right)} & \cdots & K_{n}^{\left(f_{m}, f_{m}\right)} & \overline{a_{m}} \\
a_{1} & \cdots & a_{m} & 0
\end{array}\right|}{\operatorname{det} \mathbf{K}_{n}} .
$$


Therefore, the minimum is a quadratic form in terms of the constraints and the matrix of coefficients is $\mathbf{K}_{n}^{-1}$, which is a positive definite hermitian matrix.

\section{Some applications.}

1. In [Nev2], the following problem is raised.

$$
\begin{aligned}
& \min _{\substack{p \in \mathbb{P}_{n} \\
\int p d \nu=1}}\|p\|^{2} \\
& \int p d \nu=1
\end{aligned}
$$

with $\nu$ a positive Borel measure on $[0,2 \pi)$ with finite moments. We know that the minimum is $\frac{1}{\sum_{k=0}^{n}\left|\int \varphi_{k} d \nu\right|^{2}}$ and it is attained at $\frac{\sum_{k=0}^{n} \varphi_{k}\left(\int \varphi_{k} d \nu\right)}{\sum_{k=0}^{n}\left|\int \varphi_{k} d \nu\right|^{2}}$. Besides, if we denote by $d_{n}=\left\langle z^{n}, 1\right\rangle_{\nu}$, then:

$$
\sum_{k=0}^{n} \varphi_{k}\left(\int \varphi_{k} d \nu\right)=\left(\overline{d_{0}}, \overline{d_{1}}, \quad \cdots, \overline{d_{n}}\right)\left(\begin{array}{llll}
M_{n+1}^{-1} & )^{T}(1, \quad z, & \cdots, & z^{n}
\end{array}\right)^{T}
$$

and

$$
\sum_{k=0}^{n}\left|\int \varphi_{k} d \nu\right|^{2}=\left(\overline{d_{0}}, \overline{d_{1}}, \quad \cdots, \overline{d_{n}}\right)\left(M_{n+1}^{-1}\right)^{T}\left(d_{0}, \quad d_{1}, \cdots, d_{n}\right)^{T} .
$$

2. In [ $\mathrm{GrSz}$, Chapter 10, Section 9] the prediction of a stationary discrete-time stochastic process $m$ units of time ahead is considered. The best predictor is constructed either from the moving-average representation of the process or from the spectral representation.

Here, we will present an alternative approach using the following extremal problem:

$$
\begin{gathered}
\min \\
p \in \mathbb{P}_{n} \\
p(0)=1, p^{\prime}(0)=0, \cdots, p^{(m-1)}(0)=0, m>1 .
\end{gathered}
$$

From Theorem 7 , taking into account (12) we obtain as solution of it:

$$
\tilde{\psi}_{n, 1}(z)=\frac{\left|\begin{array}{ccc}
K_{n}(z, 0) & \cdots & K_{n}^{(0, m-1)}(z, 0) \\
K_{n}^{(1,0)}(0,0) & \cdots & K_{n}^{(1, m-1)}(0,0) \\
\vdots & \vdots & \vdots \\
K_{n}^{(m-1,0)}(0,0) & \cdots & K_{n}^{(m-1, m-1)}(0,0)
\end{array}\right|}{\left|\begin{array}{ccc}
K_{n}(0,0) & \cdots & K_{n}^{(0, m-1)}(0,0) \\
\vdots & \vdots & \vdots \\
K_{n}^{(m-1,0)}(0,0) & \cdots & K_{n}^{(m-1, m-1)}(0,0)
\end{array}\right|}
$$

Notice that the best predictor can be easily deduced in terms of the coefficients of $\tilde{\psi}_{n, 1}(z)-1$. 
In the same way, from (10), we obtain an explicit expression for the minimum error of prediction:

$$
\left\|\tilde{\psi}_{n, 1}\right\|^{2}=\frac{\left|\begin{array}{ccc}
K_{n}^{(1,1)}(0,0) & \cdots & K_{n}^{(1, m-1)}(0,0) \\
\vdots & \vdots & \vdots \\
K_{n}^{(m-1,1)}(0,0) & \cdots & K_{n}^{(m-1, m-1)}(0,0)
\end{array}\right|}{\left|\begin{array}{ccc}
K_{n}(0,0) & \cdots & K_{n}^{(0, m-1)}(0,0) \\
\vdots & \vdots & \vdots \\
K_{n}^{(m-1,0)}(0,0) & \cdots & K_{n}^{(m-1, m-1)}(0,0)
\end{array}\right|}
$$

3. G. Freud showed in ([Fre]) that if $f$ is a real linear functional on the space of all polynomials, then

$$
\min _{\operatorname{deg}(P) \leq n-1} \frac{\int P^{2} d \sigma}{f(P)^{2}}=\frac{1}{\sum_{j=0}^{n-1} f\left(p_{j}\right)^{2}},
$$

and the minimum is attained by

$$
P:=\sum_{j=0}^{n-1} f\left(p_{j}\right) p_{j}
$$

Here $\left\{p_{j}\right\}$ are the orthonormal polynomials for the positive measure $d \sigma$. Freud went onto use this with $f(P)=P^{\prime}$ to establish Markov-Bernstein inequalities.

ACKNOWLEDGEMENTS. We are very grateful to the referee for pointing out the reference by G. Freud ([Fre]).

\section{REFERENCES}

[Aro] N. Aronszjan, Theory of Reproducing Kernels, Trans. Amer. Math. Soc., 68 (1950), pp. 337-404.

[Be] E. BERRIOCHOA, Productos escalares con derivadas y modificaciones a través de formas lineales, Doctoral Dissertation, Universidad de Vigo, 1997.

[Fre] G. Freud, On Markov-Bernstein type inequalities and their applications, J. Approx. Th., 19 (1977), pp. 22-37.

[GrRo] U. Grenander and M. Rosenblatt, An extension of a theorem of G. Szegö and its application to the study of stochastic processes, Trans. Amer. Math. Soc., 76 (1954), pp. 112-126.

[GrSz] U. Grenander And G. Szegö, Toeplitz Forms and Their Applications, 2nd Edition, Chelsea Publishing Company, New York, 1984.

[Nev1] P. NevaI, Gèza Freud, Orthogonal polynomials and Christoffel functions: A case study, J. Approx. Th., 48 (1986), pp. 3-167.

[Nev2] P. NeVAI, Research Problems in Orthogonal Polynomials, in Approximation Theory VI, vol II, C.K. Chui, L.L. Schumaker, and J.D. Ward, eds., Academic Press, New York, 1989, pp. 449-489.

[Sze] G. Szegő, Orthogonal Polynomials, 4th edition, Amer. Math. Soc. Colloq. Publ. 23, Providence, Rhode Island, 1975.

[VAss] W. VAN ASSCHE, Analytic aspects of orthogonal polynomials, Katholieke Universiteit Leuven, Manuscript, 1993. 